\title{
Modulation of cell wall synthesis and susceptibility to vancomycin by the two-component system AirSR in Staphylococcus aureus NCTC8325
}

\author{
Haipeng Sun, Yifan Yang, Ting Xue and Baolin Sun ${ }^{*}$
}

\begin{abstract}
Background: Vancomycin has been the medication of last resort to cure infections caused by Staphylococcus aureus since the increase in the prevalence of methicillin-resistant Staphylococcus aureus (MRSA). Some strains have developed vancomycin-intermediate resistance, which is generally associated with altered expression of or mutations in some part of the two-component system (TCS), such as GraSR, VraSR, and WalKR.

Results: We deleted the AirSR TCS in S. aureus NCTC8325 and compared the resultant transcript levels with those of its parent strain using microarray analysis. The results indicated that more than 20 genes that are related to cell wall metabolism were down-regulated in the airSR mutant. The airSR mutant exhibited reduced autolysis rates and reduced viability in the presence of vancomycin. Real-time reverse transcription PCR and DNA mobility shift assays verified that AirR can directly bind to and regulate genes that function in cell wall metabolism (cap, pbp1, and dd) and autolysis (lytM).
\end{abstract}

Conclusions: AirSR acts as a positive regulator in cell wall biosynthesis and turnover in Staphylococcus aureus NCTC8325.

Keywords: Vancomycin, Two-component system, Cell wall, Autolysis

\section{Background}

Staphylococcus aureus is a major human pathogen that can cause a number of types of infections and inflammations, ranging from superficial skin infections to life-threatening toxic shock syndrome, septicemia, osteomyelitis, and endocarditis [1]. S. aureus has developed many defense mechanisms to protect itself from the human immune system and antibiotic treatment. Methicillin-resistant Staphylococcus aureus (MRSA) has been spread worldwide, rendering the entire $\beta$-lactam class of antibiotics ineffective [2]. So far, vancomycin has been the most reliable therapeutic agent against infections caused by MRSA. Vancomycin binds to $\mathrm{D}$-alanyl-D-alanine residues of the murein monomer to interfere the synthesis of bacterial cell wall [3]. The cell wall is very important for S. aureus to maintain an osmotic gradient, and a thickened cell wall is often related to increased resistance to vancomycin

\footnotetext{
*Correspondence: xueting@ustc.edu.cn; sunb@ustc.edu.cn Department of Microbiology and Immunology, School of Life Sciences, University of Science and Technology of China, Huangshan Road, Hefei, Anhui 230027, China
}

[3]. In addition to the cell wall, S. aureus is encapsulated by capsular polysaccharides, which can protect cells from phagocytosis [4].

Two-component systems (TCSs) act as a basic stimulus-response to allow organisms to sense and respond to changes in many different environmental conditions. Typical TCSs have two components, a histidine protein kinase and a response regulator. The kinase senses the environmental stimuli, autophosphorylates at a histidine residue, and transfers the phosphoryl to an aspartate residue in the response regulator. Then the regulator is active to regulate downstream genes [5]. Bioinformatics analysis indicates that $S$. aureus harbors 16 conservative TCSs. In many cases, virulence gene expression is controlled by TCSs such as the well-studied AgrAC [6,7] and SaeSR [8]. In addition to virulence control, the TCSs are involved in the regulation of biofilm formation [9], autolysis [10], heme toxin resistance [11], cell wall synthesis [12,13], capsular polysaccharide synthesis [14], and antibiotic resistance [15-17]. In S. aureus, WalKR is a well-known TCS for its role in controlling cell wall

\section{Biomed Central}

(C) 2013 Sun et al.; licensee BioMed Central Ltd. This is an open access article distributed under the terms of the Creative Commons Attribution License (http://creativecommons.org/licenses/by/2.0), which permits unrestricted use, distribution, and reproduction in any medium, provided the original work is properly cited. 
metabolism and cell survival [12]. Recently, WalKR has been reported to be involved in vancomycin resistance [18]. By introducing a point mutation of WalK, S. aureus exhibited reduced susceptibility to vancomycin [19]. The TCS VraSR, can positively modulate cell wall biosynthesis and increase resistance to vancomycin $[13,15]$. Another TCS, GraSR, can modulate vancomycin resistance partly by regulating an adjacent $\mathrm{ABC}$ transporter, VraFG [16].

Although most TCSs in S. aureus have been well studied, the function of a few TCSs remains elusive or only partially explained. AirSR (YhcSR) was first reported to be an essential TCS [20] and was involved in the regulation of the nitrate respiratory pathway [21]. Subsequently, AirSR was described as an oxygen sensing and redoxsignaling regulator [22]. A recent study demonstrated that AirSR can regulate the lac and opuCABCD operons [23]. It appears that more work is needed to address the function of this TCS. In this study, we deleted airSR in S. aureus NCTC8325 and observed that approximately 30 cell wall metabolism-associated genes were downregulated in the airSR mutant in our microarray result. After further investigation of cell wall-related phenotypes, we found that inactivation of airSR led to reduced autolysis rates and reduced viability in sub-inhibitory concentrations of vancomycin. Real-time reverse-transcription (RT) PCR verified the down-regulation of several cell wallrelated genes and the autolysin LytM. Electrophoretic mobility shift assays indicated that AirR can directly bind to the promoter regions of $c a p, d d l, p b p 1$, and $l y t M$, indicating that airSR is directly involved in cell wall biosynthesis and turnover processes and, subsequently, vancomycin susceptibility.

\section{Methods}

\section{Bacterial strains, plasmids, and growth conditions}

The bacterial strains and plasmids used in this study are listed in Table 1. Escherichia coli DH5 $\alpha$ and BL21 (DE3) were grown in lysogeny broth (LB) medium (BD, Franklin Lakes, NJ, USA) with appropriate antibiotics $(100 \mu \mathrm{g} / \mathrm{ml}$ ampicillin sodium salt or $50 \mu \mathrm{g} / \mathrm{ml}$ kanamycin sulfate). $S$. aureus and its derivative strains were grown in tryptic soy broth (TSB) medium (BD) with erythromycin $(2.5 \mu \mathrm{g} / \mathrm{ml})$ or chloramphenicol $(15 \mu \mathrm{g} / \mathrm{ml})$ when necessary.

For collecting cells from oxygen depletion conditions, anaerobic jar of $15 \mathrm{ml}$ volume was used. Briefly, overnight cultures were diluted 1:100 into anaerobic jar containing $10 \mathrm{ml}$ TSB. Resazurin was added to a final concentration of $0.0002 \%(\mathrm{w} / \mathrm{v})$ as indicator for anaerobic conditions. The jars were incubated at $37^{\circ} \mathrm{C}$ with shaking. Initially, the cultures were in red color, and after about 6 hours incubation the red faded out completely, indicating that the oxygen was completely consumed. Then cells were collected after two more hours' incubation.
Table 1 Strains and plasmids used in this study

\begin{tabular}{|c|c|c|}
\hline $\begin{array}{l}\text { Strain or } \\
\text { plasmid }\end{array}$ & Relevant genotype & $\begin{array}{l}\text { Reference } \\
\text { or source }\end{array}$ \\
\hline \multicolumn{3}{|l|}{ Strains } \\
\hline NCTC8325 & Wild-type & NARSA $^{a}$ \\
\hline RN4220 & $\begin{array}{l}\text { 8325-4 r- initial recipient for modification } \\
\text { of plasmids which are introduced into } S \text {. } \\
\text { aureus from E. coli }\end{array}$ & NARSA \\
\hline$\Delta$ airSR & 8325 airSR::ermB & This study \\
\hline CairSR & 8325 airSR::ermB pLlairSR & This study \\
\hline $\mathrm{DH} 5 \mathrm{a}$ & 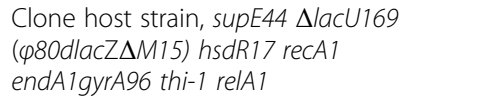 & TransGen \\
\hline BL21 (DE3) & $\begin{array}{l}\text { Express strain, } F^{-} \text {ompT hsdS }\left(r_{B}^{-} m_{B}^{-}\right) \\
\text {gal dcm(DE3) }\end{array}$ & TransGen \\
\hline \multicolumn{3}{|l|}{ Plasmids } \\
\hline $\begin{array}{l}\text { pEasy-blunt } \\
\text { simple }\end{array}$ & Clone vector, $\operatorname{Kan}^{r} \mathrm{Ap}{ }^{r b}$ & TransGen \\
\hline pET28a(+) & $\begin{array}{l}\text { Expression vector with a hexahistidine } \\
\text { tag, Kan }^{r}\end{array}$ & Novagen \\
\hline pEairR & $\begin{array}{l}\text { pET28a(+) with the airR coding sequence, } \\
\operatorname{Kan}^{r}\end{array}$ & This study \\
\hline pEairs & $\begin{array}{l}\text { pET28a(+) with the airs coding sequence, } \\
\operatorname{Kan}^{r}\end{array}$ & This study \\
\hline pEC1 & $\begin{array}{l}\text { pUC18 derivative, source of the ermB } \\
\text { gene, } A p^{r}\end{array}$ & Bruckner \\
\hline pBT2 & $\begin{array}{l}\text { Shuttle vector, temperature sensitive, } \\
\mathrm{Ap}^{r} \mathrm{Cm}^{r}\end{array}$ & Bruckner \\
\hline pBTairSR & $\begin{array}{l}\text { pBT2 containing upstream and } \\
\text { downstream fragments of airSR } \\
\text { and ermB gene, for airSR mutagenesis, } \\
\mathrm{Ap}^{r} \mathrm{Cm}^{r} \mathrm{Em}^{r}\end{array}$ & This study \\
\hline pLI50 & Shuttle cloning vector, $\mathrm{Ap}^{r} \mathrm{Cm}^{r}$ & Addgene \\
\hline pLlairSR & $\begin{array}{l}\text { pLI50 with airSR ORF and its promoter, } \\
\mathrm{Ap}^{r} \mathrm{Cm}^{r}\end{array}$ & This study \\
\hline
\end{tabular}

aARSA, Network on Antimicrobial Resistance in Staphylococcus aureus; ${ }^{\mathrm{b}} \mathrm{Kan}^{\mathrm{r}}$, kanamycin-resistant; $\mathrm{Ap}^{\mathrm{r}}$, ampicillin-resistant; $\mathrm{Cm}^{\mathrm{r}}$, chloramphenicolresistant; Emr, erythromycin-resistant.

\section{Construction of the airSR mutant and the complementary strain}

Construction of the airSR mutant strain was performed as previously described [24]. Briefly, the upstream and downstream regions of airSR were amplified from $S$. ureus NCTC8325 genomic DNA, and linked with ermB to form an up-ermB-down fragment, which was subcloned into the shuttle vector pBT2 to generate pBTairSR. The plasmid was introduced by electroporation into $S$. aureus RN4220 for modification and subsequently introduced into S. aureus NCTC8325. The strains that had allelic replacement of airSR by ermB were screened as erythromycin-resistant and chloramphenicol-sensitive colonies, and were further verified by PCR and sequencing.

For the complementation of the airSR mutation, a 2265-bp fragment of the airSR gene containing the promoter region was amplified and cloned into pLI50 to 
generate pLIairSR, which was introduced into $S$. aureus RN4220 for modification and, subsequently, introduced into the airSR mutant strain. The primers used in this study are listed in Table 2.

\section{Triton X-100-induced autolysis assay}

Triton X-100-stimulated autolysis was measured as described previously [25] with modifications. The cells (four replicates) were grown in TSB to the early exponential $\left(\mathrm{OD}_{600}=1.0\right)$ phase at $37^{\circ} \mathrm{C}$ with constant shaking $(220 \mathrm{rpm})$. The cells were collected by centrifugation, washed twice in $0.05 \mathrm{M}$ Tris- $\mathrm{HCl}$ buffer $(\mathrm{pH} 7.5)$, resuspended in an equal volume of Tris- $\mathrm{HCl}$ buffer $(0.05 \mathrm{M}, \mathrm{pH} 7.5)$ containing $0.05 \%(\mathrm{w} / \mathrm{v})$ Triton X-100 (Sigma-Aldrich, St. Louis, MO, USA), and incubated at $37^{\circ} \mathrm{C}$ with constant shaking $(220 \mathrm{rpm})$. The decrease in the optical density at $600 \mathrm{~nm}\left(\mathrm{OD}_{600}\right)$ was measured each hour using a microplate reader (Elx800, Bio-Tek, Winooski, VT, USA). The experiment was repeated at least three times with similar results.

\section{Vancomycin susceptibility assay}

For the growth experiments, overnight cultures of S. aureus were diluted to $1.0 \times 10^{7}$ colony-forming units (CFU)/ $\mathrm{ml}$ in Mueller-Hinton (MH) broth medium (BD) with or without vancomycin, and inoculated into $50 \mathrm{ml}$ flasks in a final volume of $10 \mathrm{ml}$. The flasks were incubated at $37^{\circ} \mathrm{C}$ with constant shaking $(220 \mathrm{rpm})$. The growth was monitored each hour by measuring the $\mathrm{OD}_{600}$ using a spectrophotometer (DU 730, Beckman Coulter, Brea, CA, USA). For the plate sensitivity assays, overnight cultures were collected by centrifugation and adjusted to $1.0 \times 10^{7} \mathrm{CFU} /$ $\mathrm{ml}$ with $\mathrm{MH}$. Each culture followed 4 tenfold serial dilutions, and $1 \mu \mathrm{l}$ of each sample was spotted onto a $\mathrm{MH}$ agar plate that contained 0 or $0.6 \mu \mathrm{g} / \mathrm{ml}$ of vancomycin. All the plates and cultures were incubated at $37^{\circ} \mathrm{C}$ for 24 hours before the colonies were counted. These assays were repeated at least three times with similar results.

\section{Total RNA isolation, real-time RT PCR, and microarray processing}

For the total RNA isolation, the overnight cultures of $S$. aureus were diluted 1:100 in TSB and then grown to the exponential phase until collected. The cells were processed with $1 \mathrm{ml}$ TRIzol (TaKaRa, Kyoto, Japan) in combination with 0.1-mm-diameter-silica beads in a FastPrep-24 Automated system (MP Biomedicals Solon, OH, USA), and residual DNA was removed with RNase free DNaseI (TaKaRa, Kyoto, Japan). For the reverse transcription, the cDNAs were synthesized using a PrimeScript 1st Strand cDNA Synthesis Kit (TaKaRa). The real-time PCR was performed with SYBR Premix Ex Taq (TaKaRa) using the StepOne Real-Time PCR System (Applied Biosystems, Carlsbad, CA, USA). The quantity of cDNA measured using real-time PCR was normalized to the abundance of pta cDNA [26]. The real-time PCR assays were repeated at least three times. The microarray processing and data analysis were conducted by the Biochip Company of Shanghai, China. The microarray data was uploaded to Gene Expression Omnibus (GEO) with accession number: GSE51197.

\section{Purification of AirR and Airs}

6-His-tagged AirR was cloned and purified using standard procedures. The full-length airR ORF was amplified by PCR with the e-airR-f and e-airR-r primers from $S$. aureus NCTC8325 genomic DNA, cloned into the expression vector pET28a (+) (Novagen, Merck, Darmstadt, Germany), and transformed into E. coli BL21 (DE3). The transformant was grown in $\mathrm{LB}$ at $37^{\circ} \mathrm{C}$ to an $\mathrm{OD}_{600}$ of 0.4 and induced with $0.5 \mathrm{mM}$ isopropyl- $\beta$-D-1-thiogalactopyranoside (IPTG) at $37^{\circ} \mathrm{C}$ for an additional three hours. The cells were harvested and lysed by sonication in a lysis buffer (20 mM Tris-HCl, pH 8.0, $200 \mathrm{mM} \mathrm{NaCl}$ ). The 6-His-tagged AirR protein was purified with a nickelnitrilotriacetic acid agarose solution (Qiagen, Valencia, CA, USA) following the manufacturer's recommendation. The bound protein was eluted with an elution buffer (200 mM imidazole, $20 \mathrm{mM}$ Tris- $\mathrm{HCl}$, pH 8.0, $200 \mathrm{mM} \mathrm{NaCl})$. The imidazole in the eluent was removed using a Centrifuge Biomax-5 column (Millipore, Billerica, MA, USA), and the AirR protein solution was supplemented with $30 \%$ glycerol and stored at $-80^{\circ} \mathrm{C}$ until use.

The full-length airS ORF was amplified using PCR with the e-airS-f and e-airS-r primers from S. aureus NCTC8325 genomic DNA, cloned into the expression vector pET28a (+), and transformed into E. coli BL21 (DE3). Purification of 6-His-tagged AirS was performed following the procedures of AirR purification except an overnight induction of $0.5 \mathrm{mM}$ IPTG at $16^{\circ} \mathrm{C}$. The purity of the proteins was determined by SDS-PAGE, and the protein concentration was determined using the $\mathrm{BCA}$ assay with bovine serum albumin as the standard.

\section{AirR phosphorylation in vitro}

For AirR phosphorylation in vitro, we used lithium potassium acetyl phosphate as phosphoryl group donor. Briefly, $10 \mu \mathrm{M}$ AirR was equilibrated in buffer containing $50 \mathrm{mM}$ Tris at $\mathrm{pH} 7.4,50 \mathrm{mM} \mathrm{KCl}, 5 \mathrm{mM} \mathrm{MgCl}$, and 10\% glycerol (phosphorylation buffer). Lithium potassium acetyl phosphate (Sigma-Aldrich, St. Louis, $\mathrm{MO}$, USA) was added to a final concentration of $50 \mathrm{mM}$, and this mixture was incubated for $60 \mathrm{~min}$ at $37^{\circ} \mathrm{C}$ [27].

We also used AirS for AirR phosphorylation in vitro. Briefly, $10 \mu \mathrm{l}$ phosphorylation buffer containing $2 \mu \mathrm{M}$ AirS and $10 \mathrm{mM}$ ATP was used to initiate the 
Table 2 Primers used in this study

\begin{tabular}{|c|c|c|}
\hline Primer name & Oligonucleotide $\left(5^{\prime}-3^{\prime}\right)^{a}$ & Application \\
\hline up-airSR-f & CCGgaattcTACATCTTGTGCCTTAG & airSR deletion \\
\hline up-airSR-r & ATTTGAGatcgatAATGTTCAG & airSR deletion \\
\hline down-airSR-f & CGATTTAAGTggtaccGTTGCATGATGTG & airSR deletion \\
\hline down-airSR-r & CGCggatccCCTTAAGTTGTTGGAA & airSR deletion \\
\hline Em-f & CGGatcgatGATACAAATTCCCCGTAGGC & airSR deletion \\
\hline Em-r & CGGggtaccGAAATAGATTTAAAAATTTCGC & airSR deletion \\
\hline$c$-airSR-f & CGCggatccATCGTCGCCAGTATG & $\Delta$ airS complementation \\
\hline c-airSR-r & CCGgaattcTGAAGCGAAAGTAAATG & $\Delta$ airS complementation \\
\hline e-airR-f & GGAATTCcatatgAACAAAGTAATATT & expression of AirR \\
\hline e-airR-r & CCGctcgagAATCAACTTATITTCCA & expression of AirR \\
\hline e-airs-f & GGGAATTCcatatgATGGAACAAAGGACGCGACTAG & expression of AirS \\
\hline e-airs-r & CCGctcgagCTATTTATAGGAATTGTGAATTG & expression of AirS \\
\hline RTQ-cap5B-f & GCTTATTGGTTACTTCTGA & real-time RT PCR \\
\hline RTQ-cap5B-r & GTTGGCTTACGCATATC & real-time RT PCR \\
\hline RTQ-cap5D-f & ATATGCCAGTGTGAGTGA & real-time RT PCR \\
\hline RTQ-cap5D-r & CGGTCTATTGCCTGTAAC & real-time RT PCR \\
\hline RTQ-lytM-f & CATTCGTAGATGCTCAAGGA & real-time RT PCR \\
\hline RTQ-lytM-r & CTCGCTGTGTAGTCATTGT & real-time RT PCR \\
\hline RTQ-640-f & TGATGGGACAGGAGT & real-time RT PCR \\
\hline RTQ-640-r & TATTGTGCCGCTTCT & real-time RT PCR \\
\hline RTQ-953-f & GTCATTGAGCACGATTTATT & real-time RT PCR \\
\hline RTQ-953-r & TCTGGGCGGCTGTAA & real-time RT PCR \\
\hline RTQ-pbp1-f & AGTCAGCGACCAACATT & real-time RT PCR \\
\hline RTQ-pbp1-r & AAGCACCTTCTTGAATAGC & real-time RT PCR \\
\hline RTQ-murD-f & TTCAGGAATAGAGCATAGA & real-time RT PCR \\
\hline RTQ-murD-r & AACCACCACATAACCAA & real-time RT PCR \\
\hline RTQ-1148-f & GCCGAAGTGACATAC & real-time RT PCR \\
\hline RTQ-1148-r & AAGCACCGACTGATA & real-time RT PCR \\
\hline RTQ-ddl-f & TAGGGTCAAGTGTAGGT & real-time RT PCR \\
\hline RTQ-ddl-r & GTCGCTTCAGGATAG & real-time RT PCR \\
\hline RTQ-pta-f & AAAGCGCCAGGTGCTAAATTAC & real-time RT PCR \\
\hline RTQ-pta-r & CTGGACCAACTGCATCATATCC & real-time RT PCR \\
\hline$p$-cap5A-f & TCATCTAACTCACCTGAAATTACAAAA & EMSA \\
\hline p-cap5A-r & ТTССАТТАTTТАССТСCСТTAAAAA & EMSA \\
\hline$p-d d l-f$ & CAAACTCCTITIATACTC & EMSA \\
\hline$p-d d l-r$ & GTCATTTCGTTITCCT & EMSA \\
\hline p-pbp1-f & GATTCAATAGAACAAGCGATT & EMSA \\
\hline p-pbp1-r & AGCTACACGTAATTTCGCGCTT & EMSA \\
\hline p-lytM-f & GAATCGCGAACATGGACGAA & EMSA \\
\hline p-lytM-r & GCAATCGCTGCTGCTGTTAA & EMSA \\
\hline
\end{tabular}

${ }^{\mathrm{a}}$ The sequences in lowercase letters refer to the restriction endonuclease recognition sites.

autophosphorylation of AirS. After incubation at $25^{\circ} \mathrm{C}$ for $5 \mathrm{~min}, 10 \mu \mathrm{M}$ AirR was added and the incubation was continued for another $10 \mathrm{~min}$ [22].
Electrophoretic mobility shift assay

The DNA fragments containing the promoter region were amplified from the $S$. aureus NCTC8325 genomic 
DNA. The PCR products were labeled using a digoxigenin (DIG) gel shift kit (Roche, Indianapolis, IN, USA) according to the manufacturer's instructions. The labeled fragment was incubated at $25^{\circ} \mathrm{C}$ for $15 \mathrm{~min}$ with various amounts of AirR in $10 \mu \mathrm{l}$ of incubation buffer $(10 \mathrm{mM}$ Tris-HCl, pH 8.0, $100 \mathrm{mM} \mathrm{NaCl}, 1 \mathrm{mM}$ EDTA). After incubation, the mixtures were electrophoresed in a $5 \%$ native polyacrylamide gel in $0.5 \times$ Tris-borate-EDTA (TBE) buffer. The band shifts were detected and analyzed according to the manufacturer's instructions. The images were obtained using ImageQuant LAS 4000 mini (GE, Piscataway, NJ, USA). The unlabeled fragments of each promoter were added to the labeled fragments at a ratio of approximately 50:1, respectively, as specific competitors
(SCs). The unlabeled fragments of the pta ORF region (50-fold) were added as non-specific competitors (NCs).

\section{Statistics}

The data were analyzed using the $T$-test analysis of variance, with a $P$ value of $<0.05$ considered significant (one asterisk), $P<0.01$ (two asterisks).

\section{Results}

Transcriptional profile of the airSR mutated strain

To investigate the function of AirSR, we performed a cDNA microarray analysis using total RNA from the exponential growth stage. The microarray results indicated that approximately 190 genes were up-regulated (ratio >

Table 3 Cell wall synthesis-related genes that were differentially expressed in the airSR mutant compared to the NCTC8325 wild-type

\begin{tabular}{|c|c|c|c|}
\hline Gene & & Product & $\Delta$ airSR/WT ratio ${ }^{\mathrm{a}}$ \\
\hline SAOUHSC_00114 & $\operatorname{cap} 5 A$ & Capsular polysaccharide biosynthesis protein, putative & -3.61 \\
\hline SAOUHSC_00115 & cap5B & Capsular polysaccharide synthesis enzyme Cap5B & -2.86 \\
\hline SAOUHSC_00116 & cap8C & Capsular polysaccharide synthesis enzyme Cap8C & -2.91 \\
\hline SAOUHSC_00117 & cap5D & Capsular polysaccharide biosynthesis protein Cap5D & -2.4 \\
\hline SAOUHSC_00119 & cap8F & Capsular polysaccharide synthesis enzyme Cap8F & -2.34 \\
\hline SAOUHSC_00122 & cap51 & Capsular polysaccharide biosynthesis protein Cap5I & -2.1 \\
\hline SAOUHSC_00124 & cap5K & Capsular polysaccharide biosynthesis protein Cap5K & -2.18 \\
\hline SAOUHSC_00126 & cap $8 \mathrm{M}$ & Capsular polysaccharide biosynthesis protein Cap8M & -2.02 \\
\hline SAOUHSC_00127 & $\operatorname{cap} 5 \mathrm{~N}$ & Cap5N protein/UDP-glucose 4-epimerase, putative & -2.14 \\
\hline SAOUHSC_00222 & $\operatorname{tag} B$ & TagB protein, putative & 2.24 \\
\hline SAOUHSC_00295 & $\operatorname{nan} A$ & $\mathrm{~N}$-acetylneuraminate lyase & -2.02 \\
\hline SAOUHSC_00469 & spoVG & Regulatory protein SpoVG & -2.51 \\
\hline SAOUHSC_00545 & $s d r D$ & sdrD protein, putative & -3.68 \\
\hline SAOUHSC_00640 & $\operatorname{tag} A$ & Teichoic acid biosynthesis protein & -2.08 \\
\hline SAOUHSC_00812 & CIfA & Clumping factor, ClfA & -4.72 \\
\hline SAOUHSC_00918 & & Truncated MHC class II analog protein & 2.15 \\
\hline SAOUHSC_00953 & & Diacylglycerol glucosyltransferase & -2.21 \\
\hline SAOUHSC_00974 & & Glycosyl transferase, group 1 & 4.24 \\
\hline SAOUHSC_01106 & murl & Glutamate racemase, Murl & -2.12 \\
\hline SAOUHSC_01145 & pbpl 1 & Penicillin-binding protein 1 & -2.05 \\
\hline SAOUHSC_01147 & murD & UDP-N-acetylmuramoylalanine-D-glutamate ligase, MurD & -2.58 \\
\hline SAOUHSC_01148 & $f t s Q$ & Cell division protein, putative & -2.38 \\
\hline SAOUHSC_01346 & & Glycine betaine transporter, putative & 4.62 \\
\hline SAOUHSC_01400 & & Alanine racemase, putative & -2.81 \\
\hline SAOUHSC_02317 & murF & $\begin{array}{l}\text { UDP-N-acetylmuramoylalanyl-D-glutamyl-2,6-diaminopimelate- } \\
\text { D-alanyl-D-alanyl ligase }\end{array}$ & -2.3 \\
\hline SAOUHSC_02318 & $d d l$ & D-alanyl-alanine synthetase A & -2.34 \\
\hline SAOUHSC_02399 & $\mathrm{glms}$ & Glucosamine-fructose-6-phosphate aminotransferase & -2.05 \\
\hline SAOUHSC_02444 & & Osmoprotectant transporter, BCCT family, opuD-like protein & -2.86 \\
\hline SAOUHSC_02998 & $\operatorname{cap} 5 \mathrm{C}$ & Capsular polysaccharide biosynthesis protein, Cap5C & 2.04 \\
\hline
\end{tabular}

a "_" indicates down-regulated in the airSR mutant. 
$2.0)$ and 290 genes were down-regulated (ratio $<-2.0$ ). We used Clusters of Orthologous Groups of proteins (COGs) of NCBI for protein function prediction. The expression levels of 29 cell wall metabolism-related genes were altered in the airSR mutant. The majority of these genes were down-regulated, including members of the capsular polysaccharide synthesis operon (cap operon), penicillin-binding protein 1 ( $p b p 1)$, and other enzymes that are responsible for the biosynthesis of murein sacculus and peptidoglycan. The detailed results are listed in Table 3. These data suggest that airSR plays an important role in cell wall biosynthesis.

\section{Autolysis rate induced by Triton X-100}

To test whether cell wall biosynthesis was affected, we examined Triton X-100-induced autolytic activity. The airSR mutant exhibited decreased autolysis rates compared with the wild-type strain. This phenomenon was restored by introducing a plasmid that contained the airSR operon (Figure 1), suggesting that cell wall turnover was affected by the inactivation of airSR.

\section{Viability of the airSR mutant in the presence of vancomycin}

Since vancomycin is an important antibiotic that targets $S$. aureus cell wall, we tested the viability of $S$. aureus in $\mathrm{MH}$ agar plates with vancomycin. The wild-type and the airSR mutant were able to grow at a maximum concentration of $0.6 \mu \mathrm{g} / \mathrm{ml}$ vancomycin, whereas the airSR mutant formed significantly fewer colonies (Figure 2a). We also tested cell growth in $\mathrm{MH}$ broth containing various concentrations of vancomycin. The cells were incubated in $\mathrm{MH}$ broth at an inoculum of $1 \times 10^{7} \mathrm{CFU} / \mathrm{ml}$, with constant shaking at $37^{\circ} \mathrm{C}$. No significant difference was

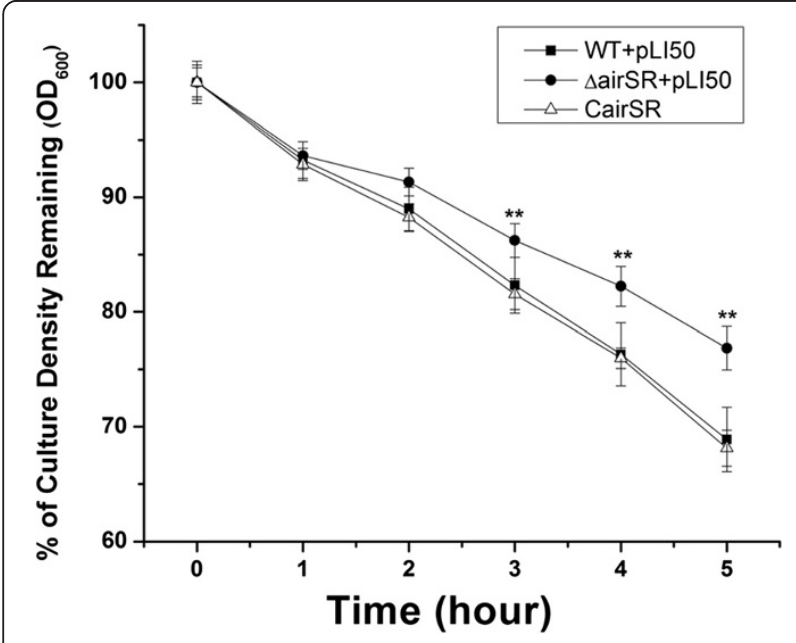

Figure 1 The Triton $\mathbf{X}-100$ induced autolysis. The wild-type, the airSR mutant, and the complementary strain in Tris- $\mathrm{HCl}$ buffer containing $0.05 \%$ Trition $\mathrm{X}-100$ at $37^{\circ} \mathrm{C}$. (**indicates $P<0.01$ ).

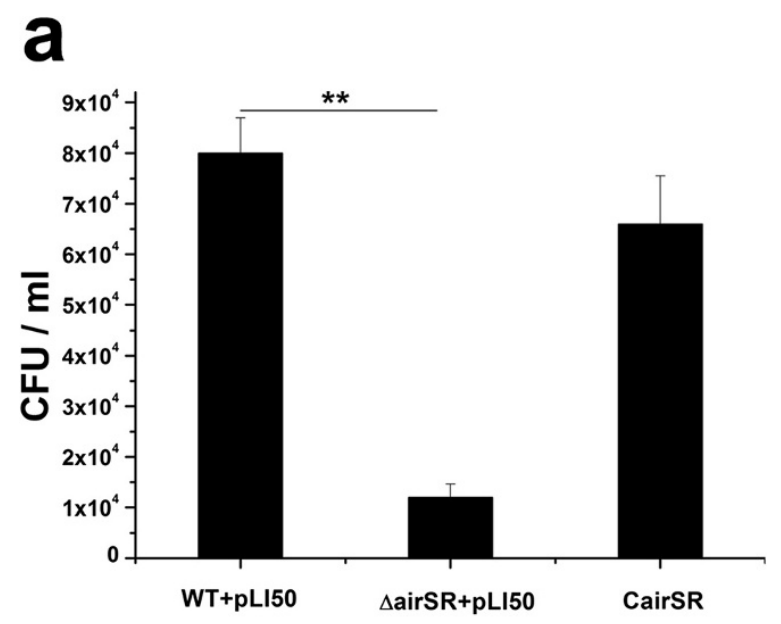

b
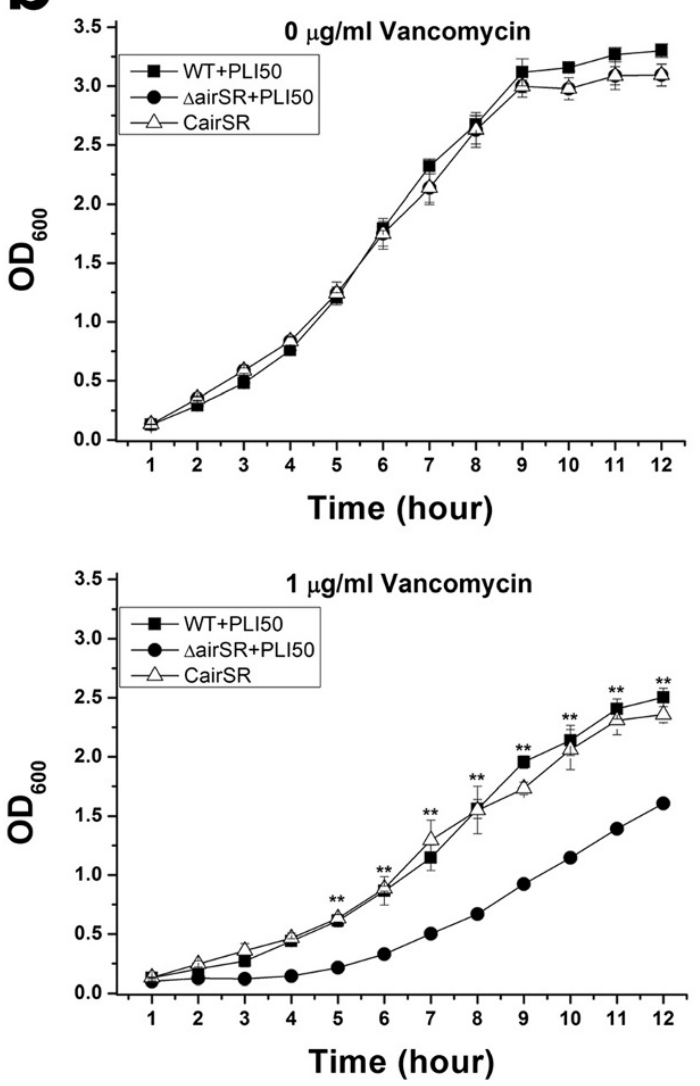

Figure 2 Vancomycin susceptibility assay. (a) Colony counts (CFU/ml) of WT, the airSR mutant, and the airSR complementary strains on $\mathrm{MH}$ agar plates containing vancomycin $(0.6 \mu \mathrm{g} / \mathrm{ml})$. The colonies were counted after incubation at $37^{\circ} \mathrm{C}$ for 24 hours. (b) The growth of the wild-type, the airSR mutant, and the airSR complementary strains in $\mathrm{MH}$ broth at $37^{\circ} \mathrm{C}$. Vancomycin concentrations of 0 or $1.0 \mu \mathrm{g} / \mathrm{ml}$. (**indicates $P<0.01$ ). 
observed when cells grew in $\mathrm{MH}$ broth without vancomycin. The airSR mutant exhibited a clear growth defect compared to the wild-type in the medium containing $1.0 \mu \mathrm{g} / \mathrm{ml}$ vancomycin (Figure $2 \mathrm{~b}$ ). Taken together, these results indicate that the airSR inactivation reduced the ability of the bacteria to survive in the presence of vancomycin.

\section{Transcriptional analysis using real-time RT PCR}

To verify the microarray results, mRNA levels from different growth stages were examined using real-time RT PCR. The mRNA levels of certain cell wall-related genes, including cap5B, cap5D, tagA, SAOUHSC_00953, pbp1, $m u r D$, fts $Q$, and $d d l$, were significantly reduced (Figure 3a, $\mathrm{b}, \mathrm{c})$. These results were in accordance with the microarray results. We also investigated the transcriptional levels of various peptidoglycan hydrolase-coding genes. Only lyt $M$ was down-regulated, as indicated by real-time PCR (Figure 3a,b,c), while atl sle 1 and $l y t N$ showed no obvious changes in expression (data not shown).

When we used cells collected from oxygen depletion conditions for real-time RT PCR, we found that only three genes (lytM, murD, fts $Q)$ showed the same down- regulation as under aerobic conditions (Figure 3d). This may suggest that AirSR needs oxygen to exert its regulation ability.

We also compared the transcriptional level of several genes from the real-time RT PCR result and the microarray data, and found a positive correlation between the two techniques (Additional file 1).

\section{The binding of AirR to the target genes}

We cloned and purified a His-tagged AirR to perform gel shift assays. DNA probes containing the putative promoters of several target genes were amplified. A clearly shifted band of DNA was visible after incubation of AirR with DNA probes containing the cap promoter (Figure 4a). The intensity of the shifted band increased as the amount of AirR was higher. This shifted band disappeared in the presence of an approximately 50-fold excess of unlabeled cap promoter DNA but not in the presence of 50-fold excess of an unlabeled coding sequence DNA of pta. These data suggest that AirR can specifically bind to the cap promoter region.

Similar assays were performed using DNA fragments of the promoter region of $d d l$ and $p b p 1$, two other genes
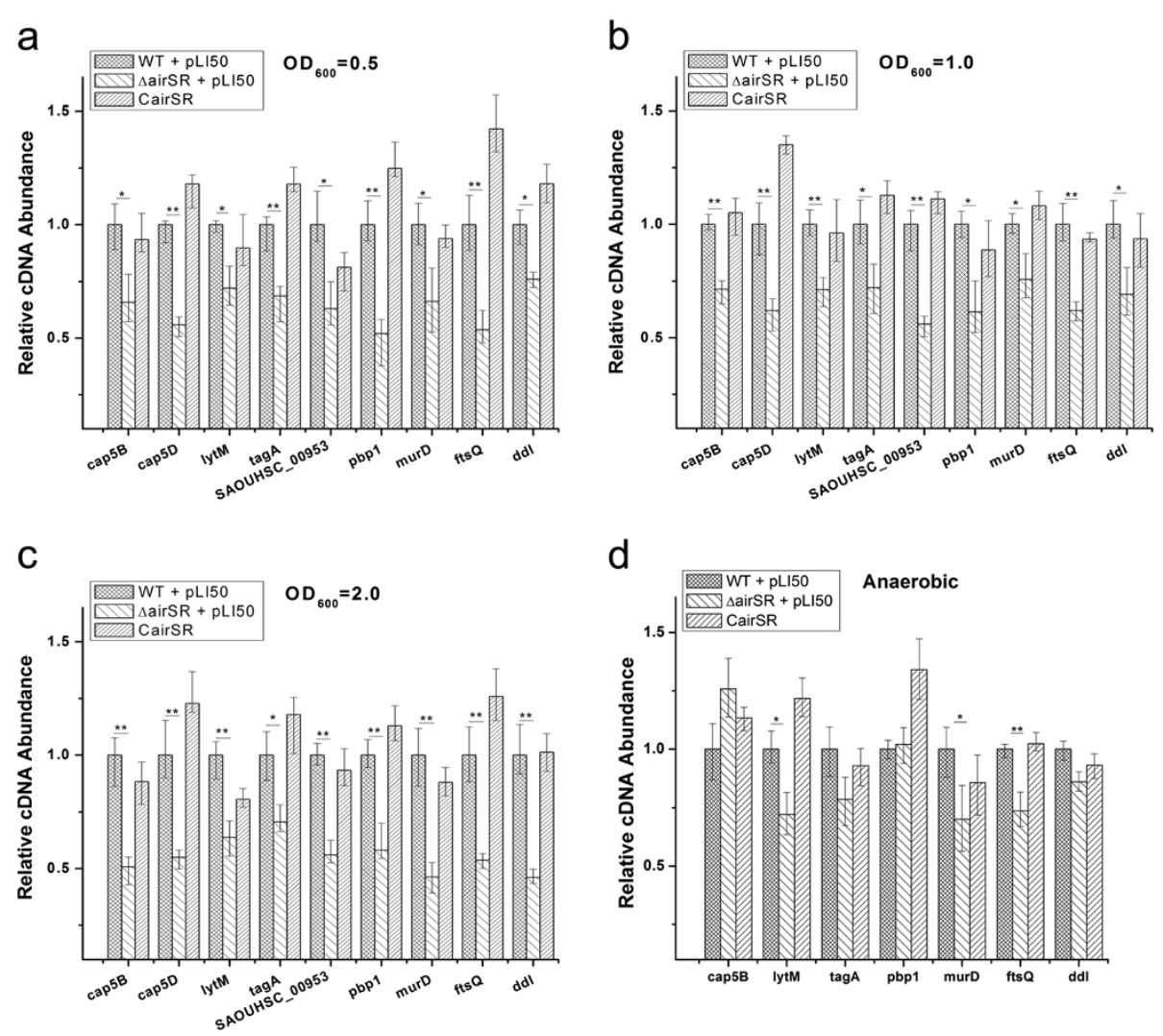

Figure 3 Transcriptional level of several cell wall-related genes. Comparison of the relative transcription levels of several cell wall biosynthesis- and hydrolysis-related genes in the wild-type, the airSR mutant, and the airSR complementary strains. (a), (b), and (c) transcriptional levels under aerobic conditions in different time courses; (d) transcriptional levels under anaerobic conditions. (*indicates $\mathrm{P}<0.05$; **indicates $P<0.01$ ). 


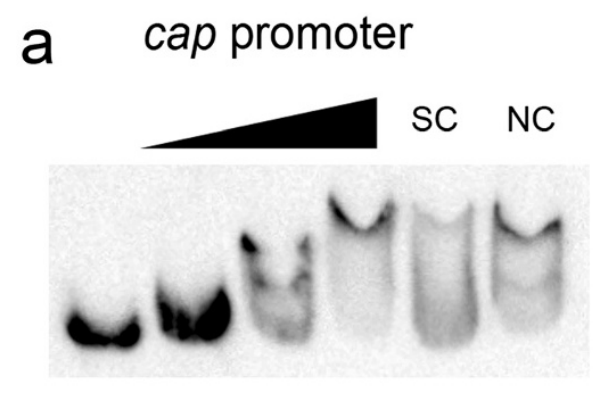

C $\quad$ pbp1 promoter

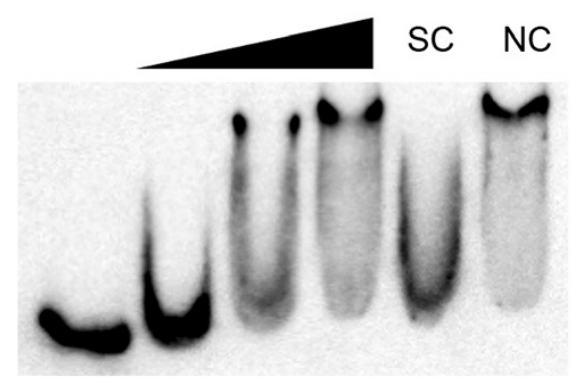

b ddl promoter

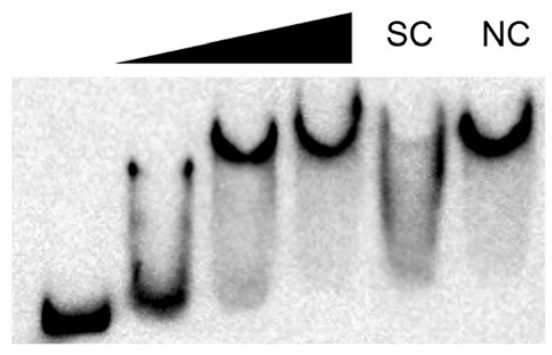

d IytM promoter

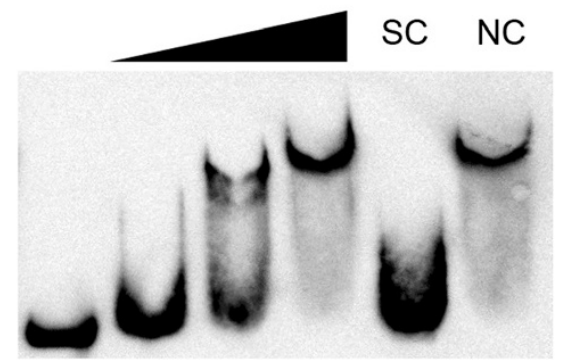

Figure 4 Electrophoretic mobility shift assay for AirR. The first lane was the free DNA probe $(2 \mathrm{nM})$; the second to fourth lanes were the DNA probe with increasing amounts of AirR $(0.3,0.6$, and $1.2 \mu \mathrm{M})$; the fifth lane was the same as the fourth lane but with the addition of a 50 -fold excess of unlabeled probes as specific competitors (SCS). The sixth lane was as the same as the fourth lane but with the addition of a 50-fold excess of unlabeled pta ORF region fragments as non-specific competitor. (NC). (a) EMSA with cap promoter; (b) ddl promoter; (c) pbp1 promoter; (d) lytM promoter.

that encode cell wall biosynthesis-related proteins. Similar promoter DNA band shift patterns were observed with the $d d l$ and $p b p 1$ promoters (Figure $4 \mathrm{~b}, \mathrm{c}$ ), suggesting that AirR can bind to these promoters. The promoter region of lyt $M$ was amplified and used as a gel shift probe. The result indicated that AirR can specifically bind to the lytM promoter (Figure $4 \mathrm{~d}$ ).

To test the effect of phosphorylation of AirR, same amount of AirR or AirR-P obtained from both lithium potassium acetyl phosphate and AirS were used for EMSA of cap promoter. The shift band from different proteins did not show obvious difference (Additional file 2), which is consistent with the observation by another group [23].

\section{Discussion}

Our study shows a direct connection between cell wall metabolism and AirSR. More than 20 genes that are related to cell wall metabolism were down-regulated in the airSR mutant, as shown by microarray analysis. Real-time RT PCR experiments confirmed the transcript level changes of several genes (cap5B, cap5D, tagA, SAOUHSC_00953, pbp1, $m u r D, f t s Q$, and $d d l)$. Real-time RT PCR indicated that the transcription of a major autolysin, LytM, was downregulated in the airSR mutant. This result is consistent with the observation of a decreased autolysis rates induced by Triton X-100 in the airSR mutant. The gel-shift assays indicated that AirR can directly bind to the promoter regions of cap, $d d l, p b p 1$, and lytM. These results suggest that AirSR enhances cell wall synthesis and degradation.

We performed the phylogenetic footprinting using promoter sequences from orthologous target genes in Staphylococci. Analysis of these sequences using CLUSTAL Multiple Sequence alignment and MEME [28] suggests that a motif "AAATNNAAAATNNNNTT" may represent the binding sequence of AirR (see Additional file 3). In our further study, we will use footprinting to identify the exact binding sequence and motif and then search genome wide for more potential targets.

Cell wall synthesis is crucial for bacterial division and growth, and it is a very important target of antibiotics, such as penicillin, vancomycin, and teicoplanin. With the increase in the number of MRSA strains, vancomycin has become the first choice to treat staphylococcal infections. The use of vancomycin has led to the emergence of vancomycin-intermediate Staphylococcus aureus (VISA). Typically, VISA exhibits thick cell walls and reduced autolysis rates. Our study demonstrated that the airSR mutation exhibited both reduced viability in vancomycin and attenuated autolysis. We speculated that, the affected 
expression of cell wall metabolism-related genes owing to the airSR mutation caused the reduction in cell viability due to vancomycin. Attenuated autolysis may be a compensatory mechanism for the affected cell wall synthesis. The reduction of viability in the presence of vancomycin and the attenuation of autolysis are two independent outcomes of the airSR mutation.

One other research group previously designated airSR as $y h c S R$ and reported that it was an essential TCS [20]. However, there are reports of an airSR mutation in several strains including Newman [22], MW2 [29], a clinically isolated strain 15981 [9], and NCTC8325, indicating that AirSR is unlikely to be essential in all strain backgrounds. Early research on airSR reported that this TCS is involved in the regulation of the nitrate respiratory pathway [21] or in the direct regulation of the lac and opuCABCD operons [23]. Our microarray results indicated the down-regulation of the nar and nre operons in the airSR mutant, which is consistent with the report that airSR can positively regulate the nitrate respiratory pathway [21]. Our microarray data, however, did not show that airSR can regulate lac or opuC operons (data not shown). Another group that first named this TCS airSR described airSR as an oxygen sensing and redoxsignaling regulator. Though they stated that airS contains a Fe-S-cluster essential for oxygen sensing and is only active in the presence of oxygen in vitro, they found that the airR mutant only affects gene expression under anaerobic conditions in strain Newman [22]. In contrast, our results showed that the expression of cell wall metabolism-related genes was not changed under anaerobic conditions (Figure 3d), but only under aerobic conditions (Figure 3a,b,c). After further comparison of their microarray data with ours, we found that, cap operon, $p b p 1$, and lytM were under negative control of AirR in their strain but positive control in our strain; saeSR, agr, and RNAIII were under negative control in their strain but not changed in our strain; spa and $h \operatorname{lgC}$ are under positive control of AirR in both strains (see Additional file 4). The discrepancy may suggest that the regulatory activity of AirR is strain specific. Why AirSR acts differently in different strains is still not clear. Our speculation is that inactivation of sigma B in NCTC8325 may contribute to the different activity of AirSR in NCTC8325 and Newman. But this speculation needs further study.

WalKR is an important TCS that positively controls cell wall biosynthesis and turnover, including directly controlling lytM [12]. Alterations in the expression of WalKR as well as WalKR mutations at amino acid sequence can lead to a change in susceptibility to vancomycin $[19,30]$. AirSR and WalKR exhibit similar functions. Our microarray data indicate that the WalKR expression level is unchanged in the airSR mutant, and there is no report so far that WalKR regulates AirSR, suggesting that the two TCSs may regulate cell wall biosynthesis independently.

\section{Conclusions}

The airSR mutant exhibited reduced autolysis and downregulation in many cell wall metabolism-related genes in $S$. aureus NCTC8325. And AirR can directly bind to the promoter region of some of these cell wall metabolism genes. These findings demonstrate that AirSR is a positive regulator in cell wall biosynthesis and turnover in $S$. aureus NCTC8325. The airSR mutant exhibited reduced viability in the presence of vancomycin, suggesting that AirSR could be a new target for controlling S. aureus infection.

\section{Additional files}

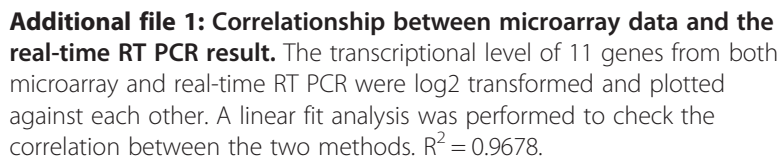

Additional file 1: Correlationship between microarray data and the real-time RT PCR result. The transcriptional level of 11 genes from both microarray and real-time RT PCR were log2 transformed and plotted against each other. A linear fit analysis was performed to check the correlation between the two methods. $R^{2}=0.9678$.

Additional file 2: EMSA of cap promoter with unphosphorylated and phosphorylated AirR. The first lane was the free DNA probe (2 $\mathrm{nM})$; the second to fourth lanes were the DNA probe with increasing amounts of unphosphorylated AirR $(0.25,0.5$, and $1 \mu \mathrm{M})$; the fifth to seventh lanes were the DNA probe with increasing amounts of lithium potassium acetyl phosphate phosphorylated $\operatorname{AirR}(0.25,0.5$, and $1 \mu \mathrm{M})$; the eighth to tenth lanes were the DNA probe with increasing amounts of AirS phosphorylated AirR $(0.25,0.5$, and $1 \mu \mathrm{M})$.

Additional file 3: Phylogenetic footprinting of AirR binding sequences. The sequences of orthologous target genes were analyzed by CLUSTAL Multiple Sequence alignment and MEME. Potential binding sequence of AirR was listed below.

Additional file 4: Comparison of microarray result of previous report. The table contains both microarray data and the verification result of real-time RT PCR.

\section{Competing interests}

The authors declare that they have no competing interests.

\section{Authors' contributions}

HS, TX, and BS designed the study. HS and YY performed laboratory work HS, YY, and TX performed data analysis. HS and YY wrote the manuscript. TX and BS critically revised the manuscript. All authors read and approved the final manuscript.

\section{Acknowledgments}

The authors thank the Network on Antimicrobial Resistance in Staphylococcus aureus (NARSA) for providing the bacterial strains. This study was supported by the National Natural Science Foundation of China (grants 31070116 and 81371850).

Received: 24 June 2013 Accepted: 4 December 2013

Published: 10 December 2013

\section{References}

1. Lowy FD: Staphylococcus aureus infections. N Engl J Med 1998, 339(8):520-532.

2. Diep BA, Otto M: The role of virulence determinants in communityassociated MRSA pathogenesis. Trends Microbiol 2008, 16(8):361-369.

3. Hiramatsu K: Vancomycin-resistant Staphylococcus aureus: a new model of antibiotic resistance. Lancet Infect Dis 2001, 1(3):147-155.

4. O'Riordan K, Lee JC: Staphylococcus aureus capsular polysaccharides. Clin Microbiol Rev 2004, 17(1):218-234. 
5. Stock AM, Robinson VL, Goudreau PN: Two-component signal transduction. Annu Rev Biochem 2000, 69:183-215.

6. Queck SY, Jameson-Lee M, Villaruz AE, Bach TH, Khan BA, Sturdevant DE, Ricklefs SM, Li M, Otto M: RNAlll-independent target gene control by the agr quorum-sensing system: insight into the evolution of virulence regulation in Staphylococcus aureus. Mol Cell 2008, 32(1):150-158.

7. Novick RP: Autoinduction and signal transduction in the regulation of staphylococcal virulence. Mol Microbiol 2003, 48(6):1429-1449.

8. Li D, Cheung A: Repression of hla by rot is dependent on sae in Staphylococcus aureus. Infect Immun 2008, 76(3):1068-1075.

9. Toledo-Arana A, Merino N, Vergara-Irigaray M, Debarbouille M, Penades JR, Lasa I: Staphylococcus aureus develops an alternative, ica-independent biofilm in the absence of the arlRS two-component system. J Bacteriol 2005, 187(15):5318-5329.

10. Brunskill EW, Bayles KW: Identification and molecular characterization of a putative regulatory locus that affects autolysis in Staphylococcus aureus. J Bacteriol 1996, 178(3):611-618.

11. Torres VJ, Stauff DL, Pishchany G, Bezbradica JS, Gordy LE, Iturregui J, Anderson KL, Dunman PM, Joyce S, Skaar EP: A Staphylococcus aureus regulatory system that responds to host heme and modulates virulence. Cell Host Microbe 2007, 1(2):109-119.

12. Dubrac S, Boneca IG, Poupel O, Msadek T: New insights into the WaIK/ WaIR (YycG/YycF) essential signal transduction pathway reveal a major role in controlling cell wall metabolism and biofilm formation in Staphylococcus aureus. J Bacteriol 2007, 189(22):8257-8269.

13. Kuroda M, Kuroda H, Oshima T, Takeuchi F, Mori H, Hiramatsu K: Twocomponent system VraSR positively modulates the regulation of cellwall biosynthesis pathway in Staphylococcus aureus. Mol Microbio/ 2003 49(3):807-821.

14. Xue T, You Y, Hong D, Sun H, Sun B: The Staphylococcus aureus KdpDE two-component system couples extracellular $\mathrm{K}+$ sensing and Agr signaling to infection programming. Infect Immun 2011, 79(6):2154-2167.

15. Gardete S, Wu SW, Gill S, Tomasz A: Role of VraSR in antibiotic resistance and antibiotic-induced stress response in Staphylococcus aureus. Antimicrob Agents Chemother 2006, 50(10):3424-3434

16. Meehl M, Herbert S, Gotz F, Cheung A: Interaction of the GraRS twocomponent system with the VraFG ABC transporter to support vancomycin-intermediate resistance in Staphylococcus aureus. Antimicrob Agents Chemother 2007, 51(8):2679-2689.

17. Hiron A, Falord M, Valle J, Debarbouille M, Msadek T: Bacitracin and nisin resistance in Staphylococcus aureus: a novel pathway involving the BraS/BraR two-component system (SA2417/SA2418) and both the BraD/ BraE and VraD/VraE ABC transporters. Mol Microbiol 2011, 81(3):602-622.

18. Howden BP, McEvoy CR, Allen DL, Chua K, Gao W, Harrison PF, Bell J, Coombs G, Bennett-Wood V, Porter JL, et al: Evolution of multidrug resistance during staphylococcus aureus infection involves mutation of the essential Two component regulator WalKR. PLoS Pathog 2011 7(11):e1002359.

19. Shoji M, Cui L, lizuka $R$, Komoto $A$, Neoh HM, Watanabe $Y$, Hishinuma $T$, Hiramatsu K: WalK and clpP mutations confer reduced vancomycin susceptibility in Staphylococcus aureus. Antimicrob Agents Chemother 2011, 55(8):3870-3881.

20. Sun J, Zheng L, Landwehr C, Yang J, Ji Y: Identification of a novel essential two-component signal transduction system, YhcSR, in Staphylococcus aureus. J Bacterio/ 2005, 187(22):7876-7880.

21. Yan M, Yu C, Yang J, Ji Y: The essential two-component system YhcSR is involved in regulation of the nitrate respiratory pathway of Staphylococcus aureus. J Bacterio/ 2011, 193(8):1799-1805.

22. Sun $F$, Ji Q, Jones MB, Deng $X$, Liang $H$, Frank B, Telser J, Peterson SN, Bae T, He C: AirSR, a [2Fe-2S] cluster-containing Two-component system, mediates global oxygen sensing and redox signaling in staphylococcus aureus. J Am Chem Soc 2012, 134(1):305-314

23. Yan M, Hall JW, Yang J, Ji Y: The essential yhcSR Two-component signal transduction system directly regulates the lac and opuCABCD operons of staphylococcus aureus. PLoS One 2012, 7(11):e50608.

24. Shang $F$, Xue $T$, Sun $H$, Xing $L$, Zhang $S$, Yang $Z$, Zhang $L$, Sun B: The Staphylococcus aureus GGDEF domain-containing protein, GdpS, influences protein A gene expression in a cyclic diguanylic acid-independent manner. Infect Immun 2009, 77(7):2849-2856.
25. Xue T, Zhao L, Sun B: LuxS/Al-2 system is involved in antibiotic susceptibility and autolysis in Staphylococcus aureus NCTC 8325. Int J Antimicrob Agents 2013, 41(1):85-89.

26. Valihrach L, Demnerova K: Impact of normalization method on experimental outcome using RT-qPCR in Staphylococcus aureus. J Microbiol Methods 2012, 90(3):214-216.

27. Belcheva A, Verma V, Korenevsky A, Fridman M, Kumar K, Golemi-Kotra D: Roles of DNA sequence and sigma a factor in transcription of the vraSR operon. J Bacteriol 2012, 194(1):61-71.

28. Bailey $T L$, Elkan C: Fitting a mixture model by expectation maximization to discover motifs in biopolymers. Proceedings/international conference on intelligent systems for molecular biology; ISMB international conference on intelligent systems for. Mol Biol 1994, 2:28-36.

29. Matsuo M, Kato F, Oogai Y, Kawai T, Sugai M, Komatsuzawa H: Distinct twocomponent systems in methicillin-resistant Staphylococcus aureus can change the susceptibility to antimicrobial agents. J Antimicrob Chemother 2010, 65(7):1536-1537.

30. Jansen A, Turck M, Szekat C, Nagel M, Clever I, Bierbaum G: Role of insertion elements and yycFG in the development of decreased susceptibility to vancomycin in Staphylococcus aureus. Int J Med Microbiol 2007, 297(4):205-215.

\section{doi:10.1186/1471-2180-13-286}

Cite this article as: Sun et al:: Modulation of cell wall synthesis and susceptibility to vancomycin by the two-component system AirSR in Staphylococcus aureus NCTC8325. BMC Microbiology 2013 13:286.

\section{Submit your next manuscript to BioMed Central and take full advantage of:}

- Convenient online submission

- Thorough peer review

- No space constraints or color figure charges

- Immediate publication on acceptance

- Inclusion in PubMed, CAS, Scopus and Google Scholar

- Research which is freely available for redistribution 